Introduction Pathogen-related diarrhoea causes significant morbidity and mortality worldwide. Our studies have previously shown that soluble dietary fibre (non-starch polysaccharides, NSP), particularly from plantain bananas, can inhibit epithelial adherence of diarrhoeal pathogens in vitro and ex vivo (J. Nutr. Biochem.2013;24:97-103).

Methods Here we aimed to establish whether plantain NSP exerts its inhibitory effect on pathogen adhesion to intestinal epithelium through either interaction with bacterial carbohydrate-binding proteins (adhesins) or via action on the epithelium itself. Prior to infection (MOI 100) of Caco2 human intestinal epithelial cells with C. difficile $(2 \mathrm{~h})$, Salmonella Typhimurium LT2 (1.5 h) or ETEC $(1.5 \mathrm{~h})$, monolayers were either 1$)$ pre-treated with plantain NSP (0$10 \mathrm{mg} / \mathrm{mL}$ - a concentration that is readily achievable in vivo with dietary supplementation) for $30 \mathrm{~min}$ followed by inoculation, 2) pre-treated for $30 \mathrm{~min}$, plantain NSP removed by thorough sterile saline washes before infection, or 3) infected with bacteria that had been pre-treated with NSP for $30 \mathrm{~min}$ followed by washing to remove non-adherent soluble fibre. Following infection, non-adherent bacteria were removed by sterile washes and adherent bacteria enumerated by overnight culture colony counts.

Results Plantain NSP $(5 \mathrm{mg} / \mathrm{mL})$ significantly decreased bacterial adhesion to Caco2 (\% inhibition of adhesion for LT2 $89 \pm 5 \%$; C.difficile $92.9 \pm 2 \%$; ETEC $65.8 \pm 1 \%$; all $\mathrm{P}<0.001$ ) compared to untreated cells. When plantain NSP was added to epithelial monolayers followed by washing to remove non-adherent fibre prior to infection, bacterial adherence was still markedly reduced (LT2 $59.2 \pm$ $5 \%$ inhibition; C.difficile $59.2 \pm 5 \%$; ETEC $45.0 \pm 2 \%$, all P $<$ 0.01). Pre-incubation of bacteria with plantain NSP followed by removal with sterile washes prior to infection resulted in no significant inhibition of adhesion compared to untreated controls. Thus inhibition of bacterial adherence to the epithelium by soluble NSP is mediated via an interaction between the NSP and the epithelium. This is supported by data from Ussing chamber experiments (PLoSOne;In press) showing that pre-treatment of human ileal tissue with plantain NSP results in a marked increase in transmucosal short circuit current $\left(\mathrm{I}_{\mathrm{sc}}\right.$ ) implying $\mathrm{Cl}^{-}$secretion (peak $\Delta \mathrm{I}_{\mathrm{sc}} 5.86 \pm 1.89 \mu \mathrm{A} . \mathrm{cm}^{-2}$ 50 min post-NSP addition; $\mathrm{P}<0.01$ ) without any effect on TEER. Conclusion Soluble plantain NSP exerts its inhibitory effect on $C$. difficile, ETEC and Salmonella adhesion to the intestinal epithelium via action on the epithelium rather than through interaction with bacterial adhesins. This effect is probably mediated by increased epithelial $\mathrm{Cl}^{-}$secretion.

Disclosure of Interest H. Simpson Grant/research support from: Provexis plc, Bo and Vera Axe:son Johnson Foundation for Nature Medicine Limited and Arcis Biotechnology, ̊. Keita: None Declared, J. Söderholm: None Declared, J. Rhodes Consultant for: advisory boards of Atlantic, Procter and Gamble and Falk, Speaker bureau with: Has received speaking honoraria from Abbott, Falk, Ferring, Glaxo Smith Kline, Proctor and Gamble, Schering Plough, Conflict with: Holds a patent with the University of Liverpool and Provexis plc for use of a soluble fibre preparation as maintenance therapy for Crohn's disease, B. Campbell Grant/research support from: Provexis plc, Bo and Vera Axe:son Johnson Foundation for Nature Medicine Limited and Arcis Biotechnology, Speaker bureau with: Received a speaking honorarium from Amgen Inc.

\section{PTH-141 WIRELESS CAPSULE ENDOSCOPY - DIAGNOSTIC YIELD AND CLINICAL UTILITY VARIES WIDELY ACCORDING TO INDICATION}

MR Smith ${ }^{*}$ B Drinkwater, R Mahmood, P Nicolson, S Ishaq, SC Cooper. Gastroenterology, Dudley Group Hospitals NHS Foundation Trust, Dudley, UK

10.1136/gutjnl-2014-307263.587
Introduction Wireless capsule endoscopy (WCE) is a well established technique for imaging the small bowel, with an increasing clinical uptake and range of indications. We aimed to evaluate the utility of WCE, comparing the diagnostic yield of procedures by indication.

Methods We performed a retrospective analysis of all WCE procedures performed at our centre, January 2007 to March 2013.

Results A total of 293 procedures were performed in 279 patients, male $47 \%$. Median age at time of procedure was 59 (IQR 45-71). The indications were: iron deficiency anaemia (IDA)/occult GI bleeding 154 (53\%), known Crohn's disease requiring assessment 58 (20\%), abdominal pain (+/- other symptoms) 33 (11\%), overt GI bleeding $20(7 \%)$, isolated diarrhoea $10(3 \%)$, coeliac disease $6(2 \%)$, isolated weight loss $4(1 \%)$, other 8 (3\%). Of those undergoing WCE for symptoms (47; $16 \%$ ), Crohn's disease was excluded (an aim of performing the study) in 34 (72\%).

The median gastric transit time was 27 min (IQR 14-55), small bowel transit time $243 \mathrm{~min}$ (IQR 181-300). Unplanned endoscopy for failure of capsule progression was required in 8 cases (3\%). 5 procedures (2\%) failed to image the small bowel (failed to leave stomach (3), battery failure (1), poor views (1)). A prokinetic was used in $9 \%(\mathrm{n}=27)$ of procedures.

Overall the diagnostic yield was $50 \%$. Separating by indication, the diagnostic yield was highest for overt GI bleeding, 70\% overall $(\mathrm{n}=14)$, identifying both $9 / 20$ small bowel causes and $5 / 20$ in colon/upper GI tract. Yield for Crohn's disease assessment was 63\%, IDA/occult GI bleeding 46\%, abdominal pain (+/- other GI symptoms) 47\%. The diagnostic yield of WCE for abdominal pain in the absence of other symptoms or abnormal radiology/ileoscopy was only 14\%. 23 out of 27 patients evaluated for symptoms (in the absence of anaemia/known Crohn's) were discharged requiring no further investigation following a negative result.

Conclusion Wireless capsule endoscopy has a good diagnostic yield, especially for evaluating GI blood loss (overt or occult) and assessing small bowel Crohn's disease. Among highly symptomatic patients, WCE can facilitate completion of investigatory pathways and enable discharge to primary care. The utility of WCE in investigating isolated abdominal pain appears limited.

Disclosure of Interest None Declared.

\section{PTH-142 DOES DOUBLE BALLOON ENTEROSCOPY AFFECT MANAGEMENT IN PATIENTS WITH SUSPECTED SMALL BOWEL TUMOURS? EXPERIENCE FROM A SINGLE TERTIARY CENTRE}

MF Hale*, ME McAlindon, DS Sanders, R Sidhu. Gastroenterology, Royal Hallamshire Hospital, Sheffield, UK

\subsection{6/gutjnl-2014-307263.588}

Introduction The advent of small bowel capsule endoscopy (CE) has greatly improved the diagnosis of small bowel tumours. Double balloon enteroscopy (DBE) is the interventional counterpart to $\mathrm{CE}$ and has the advantage of direct visualisation of small bowel pathology, biopsy and therapeutic capability. There is however a paucity of data on the use of DBE for small bowel tumours. The aim of this study was to assess the utility of DBE for small bowel tumours and to assess its impact on the diagnostic pathway in this cohort.

Methods Data was collected prospectively on all DBE procedures performed routinely between July 2006 and December 2013 particularly for the indication of small bowel tumours. 


\begin{tabular}{ll} 
Abstract PTH-142 Figure 1 & \\
\hline Diagnosis & Number of patients \\
\hline Adenocarcinoma & 4 \\
Gastrointestinal stromal tumour & 4 \\
Carcinoid & 3 \\
Lymphoma (mantle and follicular) & 3 \\
Metastatic (melanoma and transitional cell bladder) & 3 \\
Glomus tumour & 1 \\
\hline
\end{tabular}

Patient demographics, clinical presentation, findings at DBE and subsequent follow up data were recorded. The majority of patients had a number of investigations, including radiology, prior to DBE without a firm diagnosis. $83.3 \%$ had CE prior to DBE. SPSS V18 was used to analyse the data.

Results A total of 358 DBE procedures were carried out during the stated time period. Of these procedures, 18 (5.0\%) were for the indication of suspected small bowel tumour. The majority $(57.1 \%)$ of these patients were female and the average age at the time of diagnosis was 58 years $(\mathrm{SD} \pm 12 \mathrm{y})$. Indications for performing DBE included iron deficiency anaemia 44.4\%, overt bleeding $27.8 \%$, abnormal radiology $22.2 \%$ and abdominal pain $5.6 \%$. Of those who had prior CE, CE was positive in $93.3 \%$ $(14 / 15)$ of patients with the presence of a mass lesion $(50 \%)$, stricture or ulceration (35.7\%) or the presence of blood alone $(14.3 \%)$. Anterograde DBE was performed in $88.9 \%$ whilst the remaining number had DBE via the retrograde route. Tumour was successfully identified at DBE in $78 \%(\mathrm{n}=14)$ of patients, where tattoos were placed and histology obtained. Of the 4 patients where DBE failed to reach the lesion, the diagnosis was confirmed by laparotomy in 3 patients and intra-operative endoscopy in 1 patient. The final histological diagnosis of tumours identified is shown in Figure 1, UK. $61.1 \%$ tumours were located in the jejunum, $27.8 \%$ in the ileum and $11.1 \%$ in the distal duodenum. DBE influenced ongoing management in all patients that achieved a diagnosis from the procedure.

Conclusion DBE plays a valuable role in the investigation pathway of patients with suspected small bowel tumours. The step wise approach of CE followed by DBE allows efficient use of small bowel resources.

Disclosure of Interest None Declared.

\section{PTH-143 CHARACTERISTICS AND OUTCOMES OF ACUTE INFECTIVE DIARRHOEA- A LARGE TEACHING HOSPITAL COHORT}

'S Mumtaz*, 'S Kelly, 'UA Ahmad, 'S Everett. 'Gastroenterology, Leeds Teaching Hospitals, Leeds, UK; ${ }^{2}$ Acute Medicine, Doncaster Royal Infirmary, Doncaster, UK

\subsection{6/gutjpl-2014-307263.589}

Introduction The clinical syndrome associated with infective diarrhoea is well known, however there are few studies which have rigorously analysed the characteristics in history, examination and investigation in a single cohort of patients.

Methods This was a retrospective cohort study of patients admitted to the gastroenterology department of a single tertiary care teaching hospital with a culture positive episode of bacterial infective diarrhoea, excluding Clostridium difficile. Data was collected from clinical notes of patients presenting over an 8 year period (2004 - 2012) with a follow up period of 12 months from the date of admission.

Results 103 patients were included in the study; 59 (57\%) males, mean age 43 (17-101). Organisms cultured were;
Campylobacter 84, Salmonella 13, Shigella 2, E coli 4. Mean duration of symptoms was 5.7 days (1-14) while patient gave history of; abdominal pain $88 \%$ (91), per rectal (PR) bleeding $52 \%$ (53), vomiting 43\% (44), weight loss 22\% (23), food history $27 \%(28)$, and recent travel $8.5 \%$ (9). 13 patients $(12 \%)$ had pre-existing inflammatory bowel disease (IBD) and 35 patients (34\%) were on a PPI. Examination revealed; fever (>37.5) 35\% (35), tachycardia 27\% (28) and hypotension in 4\% (5) patients. Blood test showed; elevated CRP $(>10)$ in $98 \%$ patients [median 81 (IQR 99)], high white cell count $(>11)$ in 12\% [median 7.8 (IQR 3.7)] and a raised platelet count $(>400)$ in 5\% patients [median 241 (IQR 92)]. Steroids were given to $24(23 \%)$ patients, 9 had pre-existing IBD, mean duration 3 days where as 30 patients (29\%) were given a course of antibiotics after the culture results were known. Mean length of stay was 5 days $(1-30)$; there were 3 in-patient deaths and no surgical interventions. At 12 months 1 patient was diagnosed with IBD but there were no deaths or episodes of re-infection.

Conclusion Acute onset diarrhoeal symptoms associated with abdominal pain, vomiting, or pr bleed and elevated inflammatory markers especially CRP should raise the suspicion of an infective aetiology even if the patient has pre-existing IBD. One in three patients was noted to have pyrexia but contrary to popular belief a history of travelling or food was not a common occurrence.

Disclosure of Interest None Declared.

\section{PTH-144 NATIONWIDE STUDY OF EMERGENCY ADMISSIONS TO ACUTE HOSPITALS IN ENGLAND FOR ANOREXIA NERVOSA: IMPLICATIONS FOR MARSIPAN}

${ }^{1} \mathrm{M}$ Shawihdi ${ }^{*},{ }^{2} \mathrm{E}$ Thompson, ${ }^{3} \mathrm{~S}$ Sharma, ${ }^{4} \mathrm{~S}$ Lal, ${ }^{2} \mathrm{M}$ Pearson, ${ }^{1} \mathrm{~K}$ Bodger. ${ }^{1}$ Department of Gastroenterology, Institute of Translational Medicine, University of Liverpool, Liverpool, UK; ${ }^{2}$ Aintree Health Outcomes Partnership, University of Liverpool, Liverpool, UK; ${ }^{3}$ Priory Eating Disorders Services, The Priory Hospital Cheadle Royal, Cheadle, UK; ${ }^{4}$ Gastroenterology and Intestinal Failure Unit, Salford Royal NHS Foundation Trust, Salford, UK

\subsection{6/gutjnl-2014-307263.590}

Introduction Patients with anorexia nervosa (AN) develop significant physical complications leading to emergency admission (EmAd) to acute hospitals. There are few data on national burden, institutional case volume, frequency, nature or outcomes of EmAds for this rare, complex condition. The need for joint management of AN between medical and psychiatric teams has been highlighted ${ }^{1}$. We aimed to define characteristics of adult patients with AN admitted as emergencies to acute hospitals in England. Methods A 2-year download of data for English acute hospitals (Hospital Episode Statistics) was obtained, linked to death registry. We extracted all EmAds in medical or surgical specialties containing ICD-10 codes for AN. Adult patients with a first (index) admission between Oct 07 and Sept 08 were selected (1year incident cohort). Admissions in 6 months before or after index admission were extracted and ordered chronologically. Demographics and diagnosis codes for each admission were reviewed independently by two gastroenterologists $(\mathrm{SL}, \mathrm{KB})$ and a psychiatrist (SS), selecting only cases where 2 of 3 reviewers judged the coding sequence consistent with EmAd for AN. The index EmAd was classified according to primary diagnosis.

Results 549 AN patients were admitted to 132 Trusts in England during the year. Mean age [sd]: 30 [11] yrs; Female: 95.4\%; $\geq 1$ Charlson co-morbidities: $11.3 \%$. Primary diagnosis: AN, 33.5\%; Complication of AN or a GI symptom, 39.2\%; Poisoning or Self Harm, 17.5\%; Alcohol-related, 2.4\%; Miscellaneous diagnoses, 